\title{
Glide symmetry for mode control and significant suppression of coupling in dual-strip SSPP transmission lines
}

\author{
Xiao Tian Yan $\odot,{ }^{a, \dagger}$ Wenxuan Tang, ${ }^{\mathrm{a}, \mathrm{b}, \boldsymbol{k}, \dagger}$ Jun Feng Liu $\odot,{ }^{a}$ Meng Wang, ${ }^{\mathrm{a}}$ Xin Xin Gao, ${ }^{\mathrm{a}}$ and Tie Jun Cui ${ }^{\mathrm{a}, \mathrm{b}, \star}$ \\ aSoutheast University, School of Information Science and Engineering, State Key Laboratory of Millimeter Waves, Nanjing, China

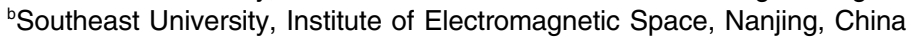

\begin{abstract}
Glide symmetry, which is one kind of higher symmetry, is introduced in a special type of plasmonic metamaterial, the transmission lines (TLs) of spoof surface plasmon polaritons (SSPPs), in order to control the dispersion characteristics and modal fields of the SSPPs. We show that the glide-symmetric TL presents merged pass bands and mode degeneracy, which lead to broad working bandwidth and extremely low coupling between neighboring TLs. Dual-conductor SSPP TLs with and without glide symmetry are arranged in parallel as two channels with very deep subwavelength separation (e.g., $\lambda_{0} / 100 \mathrm{at} 5 \mathrm{GHz}$ ) for the application of integrated circuits and systems. Mutual coupling between the hybrid channels is analyzed using coupled mode theory and characterized in terms of scattering parameters and near-field distributions. We demonstrate theoretically and experimentally that the hybrid TL array obtains significantly more suppressed crosstalk than the uniform array of two nonglide symmetric TLs. Hence, it is concluded that the glide symmetry can be adopted to flexibly design the propagation of SSPPs and benefit the development of highly compact plasmonic circuits.
\end{abstract}

Keywords: glide symmetry; spoof surface plasmon polaritons; dispersion control; mode degeneracy; coupling suppression.

Received Oct. 21, 2020; revised manuscript received Dec. 23, 2020; accepted for publication Jan. 11, 2021; published online Feb. 27, 2021.

(C) The Authors. Published by SPIE and CLP under a Creative Commons Attribution 4.0 Unported License. Distribution or reproduction of this work in whole or in part requires full attribution of the original publication, including its DOI.

[DOI: 10.1117/1.AP.3.2.026001]

\section{Introduction}

Surface plasmon polaritons (SPPs) are highly localized surface waves that exist on the interface of metal and dielectric beyond the far-infrared frequency. However, they do not naturally exist at lower frequencies such as terahertz and microwaves, where the metals no longer behave like plasma with negative dielectric constants. ${ }^{1}$ In order to allow SPPs to operate in the lower frequency bands, spoof surface plasmon polaritons (SSPPs) have been conceived and realized on periodically structured metallic surfaces. ${ }^{2}$ Similar to the optical SPPs, SSPPs exhibit highly localized electromagnetic (EM) fields, subwavelength resolution, and extraordinary field confinements. ${ }^{3}$ Furthermore, ultrathin corrugated metallic strips have been proposed as

${ }^{*}$ Address all correspondence to Wenxuan Tang, wenxuant@ seu.edu.cn; Tie Jun Cui, tjcui@seu.edu.cn

'These authors contributed equally to this work kind of slow-wave waveguide that supports the propagation of SSPPs. ${ }^{4}$ Different types of SSPP transmission lines (TLs), including single-conductor and dual-conductor ones, have been created in different circumstances. Because of mode and momentum mismatch, transitions from the conventional TLs such as coplanar waveguide and microstrip (MS) are needed to realize high-efficiency excitation of SSPPs. ${ }^{5-7}$ In addition, a series of devices, such as filters, resonators, amplifiers, demultiplexers, and antennas, has been demonstrated, ${ }^{8-13}$ and an integrated wireless communication system based on SSPPs has been recently developed..$^{14}$ The SSPP circuits are highly expected to offer new solutions for future integrated and smart systems due to the designable dispersion characteristics, high efficiency, low bending loss, low crosstalk, conformal transmissions, and low interference with EM waves in the environment. ${ }^{15-18}$

On the other hand, the SSPP structures can be considered as special kinds of metamaterials that are composed of periodically 
arranged subwavelength unit cells. It has been demonstrated that higher symmetries can effectively affect the dispersion characteristics and band gaps of structures. ${ }^{19}$ Higher symmetry occurs if the periodic structure can be described by additional geometric operations beyond a simple translation or reflection. For example, glide and twist symmetries are two common cases of higher symmetry. ${ }^{20,21}$ Most recently, metamaterials with glide symmetry have been developed to achieve flexible control of dispersion behaviors and band gaps, so as to provide new solutions for microwave waveguides, functional devices, and antennas. $^{22-24}$

Here, we introduce glide symmetry in dual-conductor SSPP TLs and TL arrays for characteristics control. First, the dispersion curves, modal fields, and transmission properties of the glide symmetric TL are studied, and degeneracy of the fundamental mode is investigated. After that, two SSPP TLs, one with glide symmetry and one without glide symmetry, are arranged in parallel as two channels with deep subwavelength separation (which is $\lambda_{0} / 100$ at $5 \mathrm{GHz}$ ). Mutual coupling between the hybrid channels is analyzed based on coupled mode theory and characterized in terms of scattering parameters and near-field distributions. Simulated and measured results are presented, showing that the hybrid TL array obtains much higher channel isolation and lower crosstalk than the uniform array composed of two nonglide symmetric TLs. Finally, conclusions are drawn and potential applications are discussed.

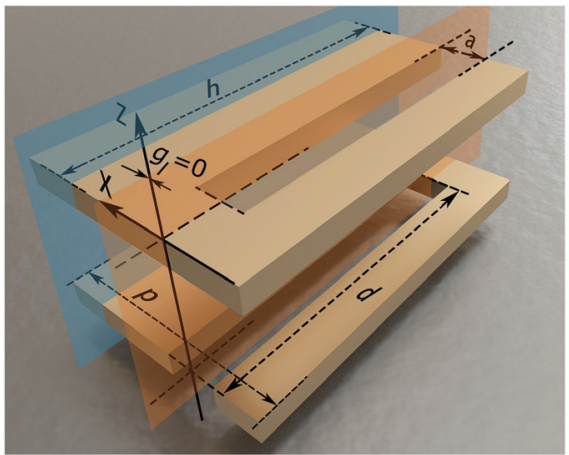

(a)

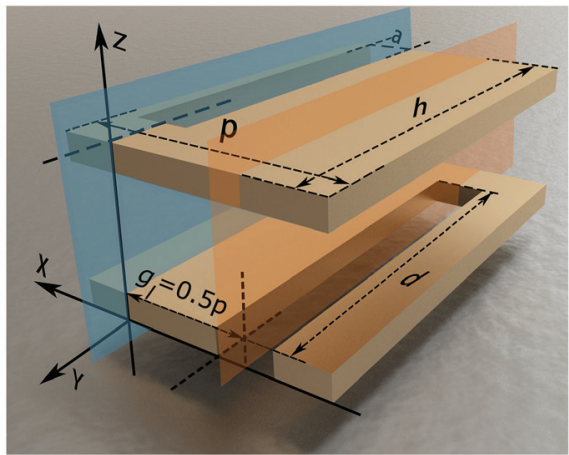

(b)

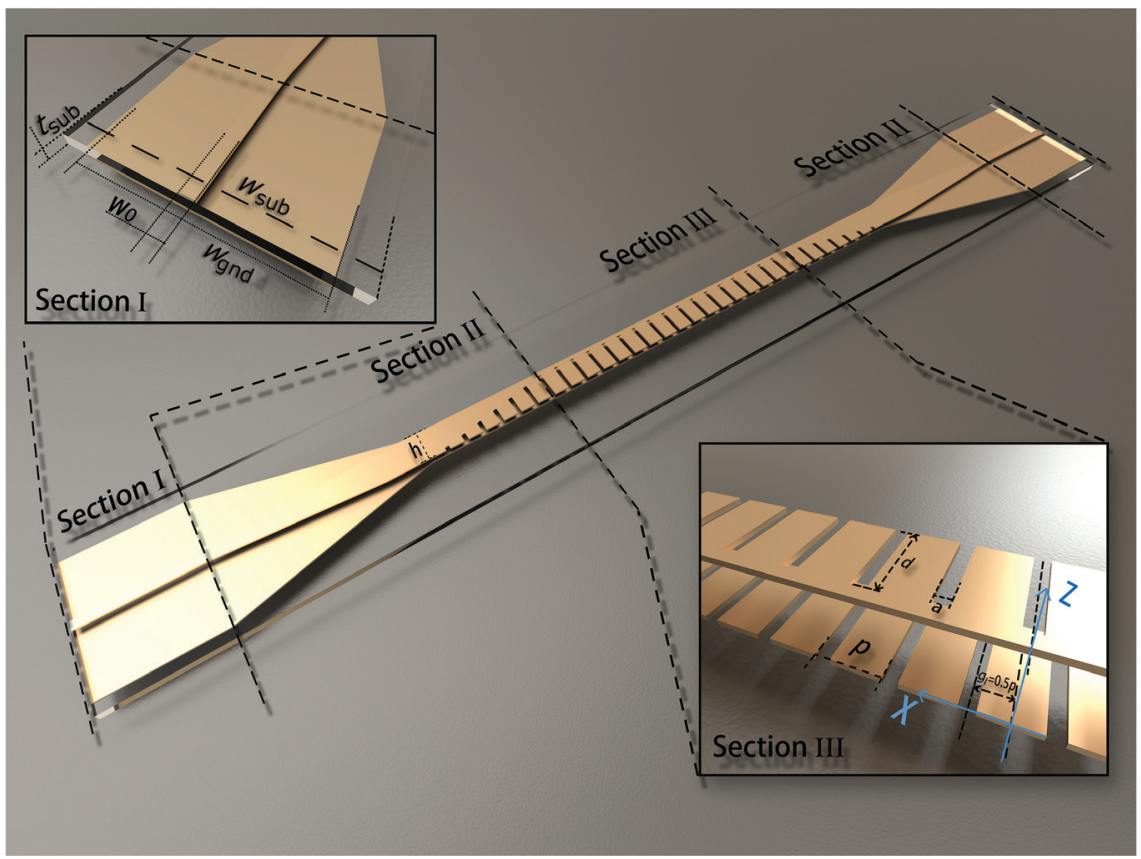

(c)

Fig. 1 (a) A nonglide unit cell in the dual-strip SSPP TL. The line width, period, width, and depth of the slots are $h=6 \mathrm{~mm}, p=3.6 \mathrm{~mm}, a=0.8 \mathrm{~mm}$, and $d=5.2 \mathrm{~mm}$, respectively. In this work, the thickness of the two metal layers is $t=0.018 \mathrm{~mm}$, and the substrate has a relative permittivity of 3.48 with thickness of $t_{\text {sub }}=0.762 \mathrm{~mm}$ and width of $w_{\text {sub }}=30 \mathrm{~mm}$. (b) A glide symmetric unit cell. The centers of the slots in the upper and lower layers are misaligned with a glide distance of $g_{l}=0.5 p$ along the $x$ axis. (c) The glide symmetric dual-strip SSPP TL. Section I is the MS line at the input and output, and the widths of the upper and lower strips are $w_{0}=1.7 \mathrm{~mm}$ and $w_{\text {gnd }}=25 \mathrm{~mm}$, respectively. Section II is the transition section and Section III is composed of uniform glide symmetric unit cells. 


\section{Glide Symmetry in Dual-Strip SSPP TLs}

The SSPP TLs typically consist of metallic strips with subwavelength corrugations and sometimes with ground. Their configurations (e.g., the geometry of the strips) can be adjusted according to specific requirements in applications. There have been several types of SSPP TLs reported in the literature, which could be generally categorized as single-strip, grounded single-strip, and dual-strip. ${ }^{4,7,8}$ Among them, the dual-strip SSPP TLs contain two layers of antisymmetrically arranged corrugated metallic strips on both sides of dielectric substrate. Compared to the single-strip SSPP TLs of the same size, the dual-strip ones obtain lower cutoff frequency, stronger subwavelength effect and field confinement, further depressed mutual coupling, and easier embedment of lumped elements and active devices. ${ }^{25}$ In view of these, we introduce glide symmetry in the dual-strip SSPP TLs to further improve the performance of plasmonic circuits.

A unit cell of the dual-strip SSPP TL, which has two antisymmetrically arranged metallic structures, is given in Fig. 1(a). For comparison, the unit cell with glide symmetry is given in Fig. 1(b). The misalignment between the upper and lower strips is indicated by the glide distance between the centers of the upper and lower slots (which is denoted as $g_{l}$ in the figure). When there is no glide symmetry introduced in the structure, the upper and lower metallic strips are aligned along the $x$ axis with $g_{l}=0$. When the glide symmetry is introduced, the misalignment is no longer zero.

To compare the EM properties of the nonglide and glide symmetric structures, dispersion characteristics are studied in the commercial software of CST Microwave Studio. The dashed lines in Fig. 2 are the dispersion curves of the first four modes of the nonglide symmetric SSPP structure. The cutoff frequency of the fundamental mode (mode 1 in Fig. 2) is about $5 \mathrm{GHz}$, indicating that the SSPP wave in this mode can propagate below $5 \mathrm{GHz}$ but becomes evanescent above this frequency. At higher frequencies, higher-mode SSPPs may also propagate according to the higher-mode dispersion curves, and stop bands are observed between neighboring propagating modes, indicating the standing waves at the Brillouin zone boundary. For the glide

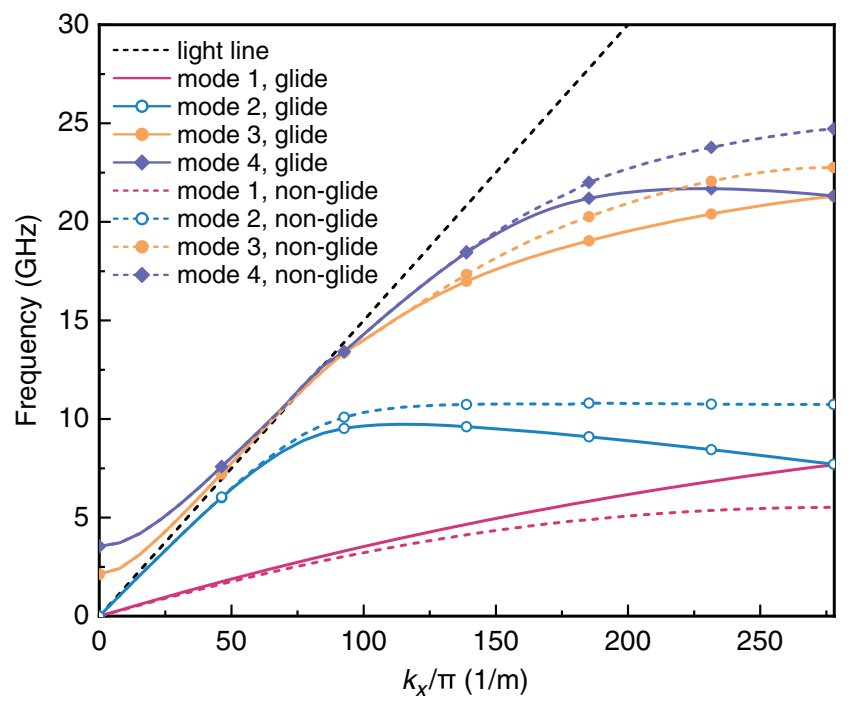

Fig. 2 Dispersion diagram of the nonglide and glide symmetric unit cells. symmetric SSPP structure, we have tested different misalignments and find that when the misalignment is half periodicity $\left(g_{l}=0.5 p\right)$ there exists a degeneracy with nonzero group velocity of the supported modes at the Brillouin zone boundary. In particular, the first and second modes, as well as the third and fourth modes, coalesce at the edge of the first zone of the Brillouin diagram, and their dispersion curves are considered as part of the same mode, as illustrated by the four solid lines in Fig. 2. The band gap between the first and second modes, and the one between the third and fourth modes, disappear in the glide symmetric structure. As a result, the cutoff frequency of the fundamental mode (the first mode) increases from 5 to $9.5 \mathrm{GHz}$, indicating that the glide symmetric SSPP TL obtains a significantly broadened working band without extra space or cost.

The degeneracy of the fundamental mode is also studied in terms of the distribution of EM fields. We choose two cross sections in the unit cell, as indicated by the orange plane and the blue one in Figs. 1(a) and 1(b), to observe the near-electric fields in the nonglide and glide symmetric SSPP structures. For the nonglide symmetric one, the electric field of the fundamental mode is nearly asymmetrically distributed in both cross sections [see Figs. 3(a) and 3(b)], which is naturally decided by the antiarrangement of the upper and lower strips. In other words, the fundamental mode of the nonglide SSPP TL is approximately an odd mode with the cutoff frequency of $5.2 \mathrm{GHz}$. Between the fundamental mode and the first higher mode (the second mode), there exists a band gap where no SSPP wave can propagate. In contrast, for the glide symmetry, there are both even and odd components included in the total field, ${ }^{26}$ and the distributions of the field become more uniform in different cross sections [e.g., the field distribution in Fig. 3(c) is similar to that in Fig. 3(d) when flipped], which is mainly due to the glide symmetric property of the structure. Meanwhile, the cutoff frequency of the fundamental mode changes to $9.5 \mathrm{GHz}$ since the first band gap is eliminated. The change of modal field and band gap is due to the degeneracy of modes when higher symmetry is introduced in the structure. We remark that the fundamental modes of the nonglide and glide symmetric SSPP TLs have essentially high isolation because of the enhanced orthogonality of EM fields. This important characteristic could be utilized in the design of compact TL arrays, as is discussed in Sec. 3.

The entire model of the glide symmetric SSPP TL is shown in Fig. 1(c). Similar to the dual-strip SSPP TL reported in Ref. 17, the glide symmetric one includes three sections: Section I contains MS lines at the input and output, Section II contains transitions between the MS lines and SSPP structures, and Section III contains uniform unit cells that support the propagation of SSPPs. In the transitions, the quasitransverse electromagnetic mode on the MS line is transformed to the TM mode of SSPPs, and vice versa. In order to realize smooth matching of impedance and momentum, the widths of the upper and lower strips ( $w_{0}$ and $w_{\text {gnd }}$, respectively) and the depth of slot (d) are changed gradually. The prototypes of nonglide and glide symmetric TLs were fabricated on a Rogers RO4350B substrate with relative permittivity of 3.48 and thickness of $0.762 \mathrm{~mm}$. The transmission coefficients (S21) and reflection coefficients (S11) of both TLs were measured using an Agilent vector network analyzer (VNA) and plotted in Fig. 4. In terms of the transmission coefficient, the nonglide symmetric TL has a cutoff frequency of about $5.4 \mathrm{GHz}$ while the glide symmetric one has 


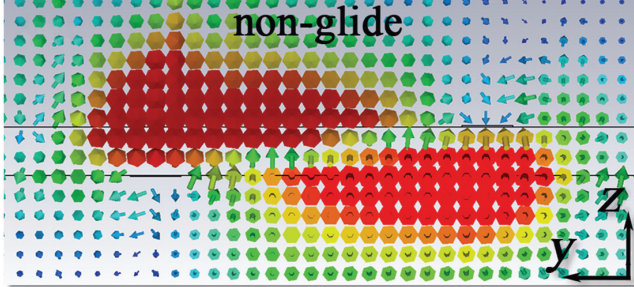

(a)

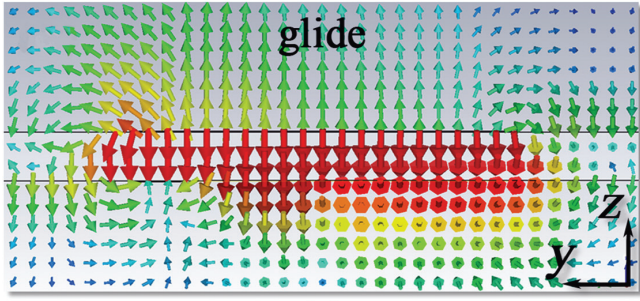

(c)

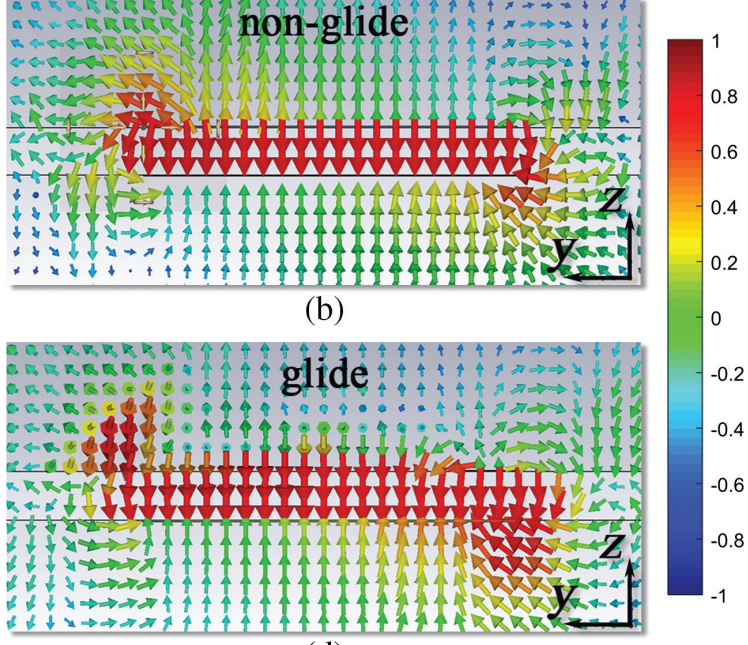

(d)

Fig. 3 (Side view) (a), (b) Distributions of the electric fields of the nonglide symmetric structure at the orange and the blue cross sections indicated in Fig. 1(a), respectively. (c), (d) Distributions of the electric fields of the glide symmetric structure at the orange and the blue cross sections indicated in Fig. 1(b), respectively.

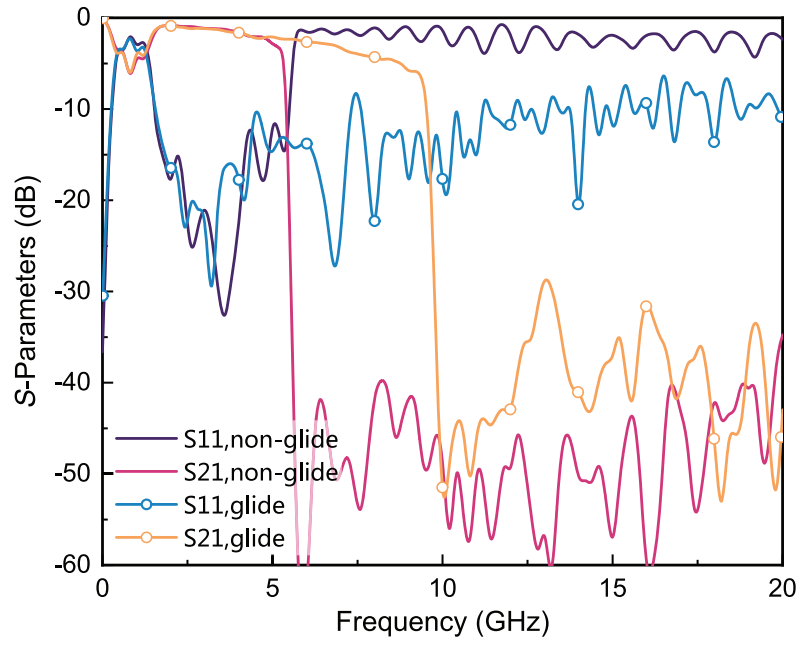

Fig. 4 Measured reflection coefficients (S11) and transmission coefficients (S21).

a cutoff frequency of about $9.6 \mathrm{GHz}$, which agrees quite well with the dispersion curves plotted in Fig. 2. Therefore, it is proved that the bandwidth could be significantly increased (almost double) for the glide symmetric SSPP TL, while the size of it remains the same as that of the nonglide one. According to the dispersion curve of the glide symmetric SSPP TL, we remark that the higher-frequency part of the fundamental mode (approximately from 5.4 to $9.6 \mathrm{GHz}$ ) is a hybrid mode including both forward harmonic (with a positive wave velocity) and backward harmonic (with a negative wave velocity). Further investigations may be carried out in the future so as to excite a purer backward harmonic for novel properties and functionalities.

The distributions of the near electric fields are measured in our home-made near-field scanning system, which is mainly constructed by a two-dimensional (2D) stepper and the VNA. The magnitudes of the electric fields right above the nonglide and glide symmetric TLs are measured and plotted in Fig. 5. For the nonglide TL, the SSPP wave propagates below the cutoff frequency of $5.4 \mathrm{GHz}$ (e.g., at $5.3 \mathrm{GHz}$ ) but becomes evanescent beyond it (e.g., at $5.9 \mathrm{GHz}$ ). In contrast, for the glide symmetric TL, the SSPP wave can propagate below the cutoff frequency of $9.6 \mathrm{GHz}$ (e.g., at $5.3,5.9$, and $8.5 \mathrm{GHz}$ ) with gradually decreased transmission efficiency.

\section{Hybrid Nonglide and Glide Symmetric TL Array}

Channel crosstalk is inevitable in compact systems, due to mutual coupling between TLs and components. When TLs are densely arranged, e.g., with subwavelength separation, the EM fields on one TL will give rise to induced currents on other TLs and therefore cause unwanted coupling. In fact, channel crosstalk suppression and signal integrity are two key problems in modern integrated circuits, and, unfortunately, they are almost contradictory. The SSPP TLs have natural advantages in view of coupling suppression, due to the strong subwavelength effect and field confinement. Here, we propose a hybrid array composed of alternatively arranged nonglide and glide symmetric TLs, which possesses extremely low mutual couplings.

A simplified two-channel model is used to investigate the isolation and coupling characteristics of the hybrid TL array, as is given in Fig. 6. This is a four-port network composed of two channels; one is the nonglide symmetric TL channel, and the other is the glide symmetric one. Each TL includes three sections, as discussed in Fig. 1(c). The size of the unit cell, dielectric substrate, and metal thickness are the same for both TLs. The four ports are named clockwise, as shown in Fig. 6. Separation between the two TLs is indicated by dis in the straight section (Section III), which is in the deep subwavelength scale.

Due to the mode mismatch, the hybrid configuration brings in a broadband suppression of near-field coupling. As discussed above, the fundamental mode of the glide symmetric TL is totally different from that of the nonglide one. According to 
nonglide-symmetric TL

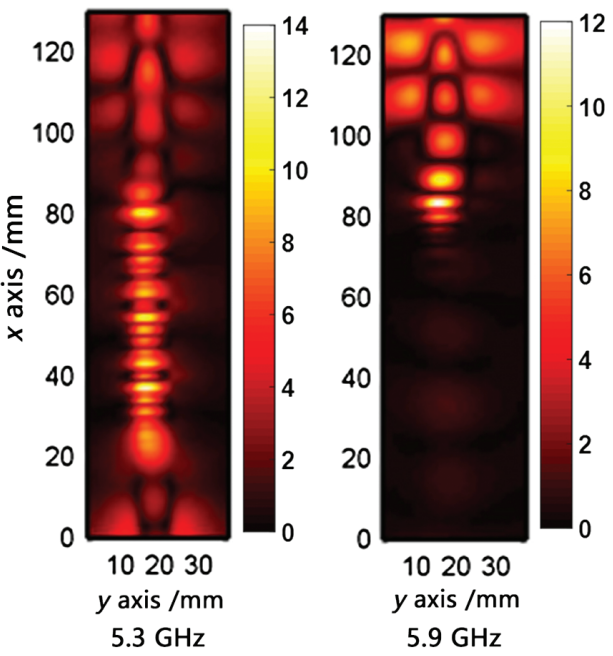

(a)

glide-symmetric TL

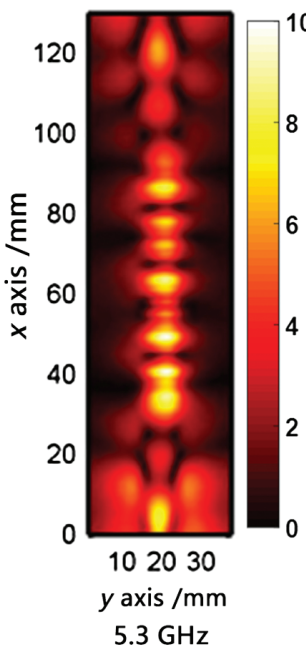

$5.3 \mathrm{GHz}$

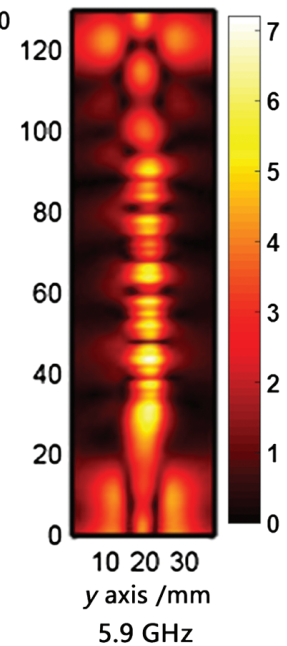

(b)

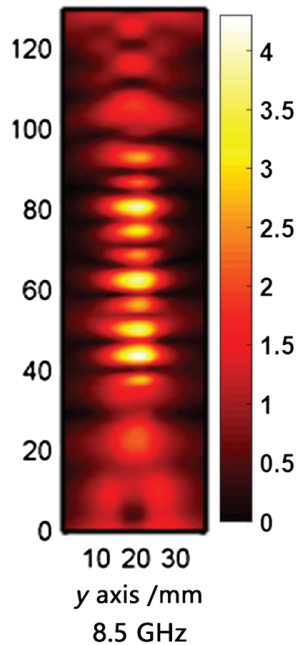

$8.5 \mathrm{GHz}$

Fig. 5 Magnitude distributions of the near electric field for (a) the nonglide symmetric and (b) the glide symmetric SSPP TLs at different frequencies.

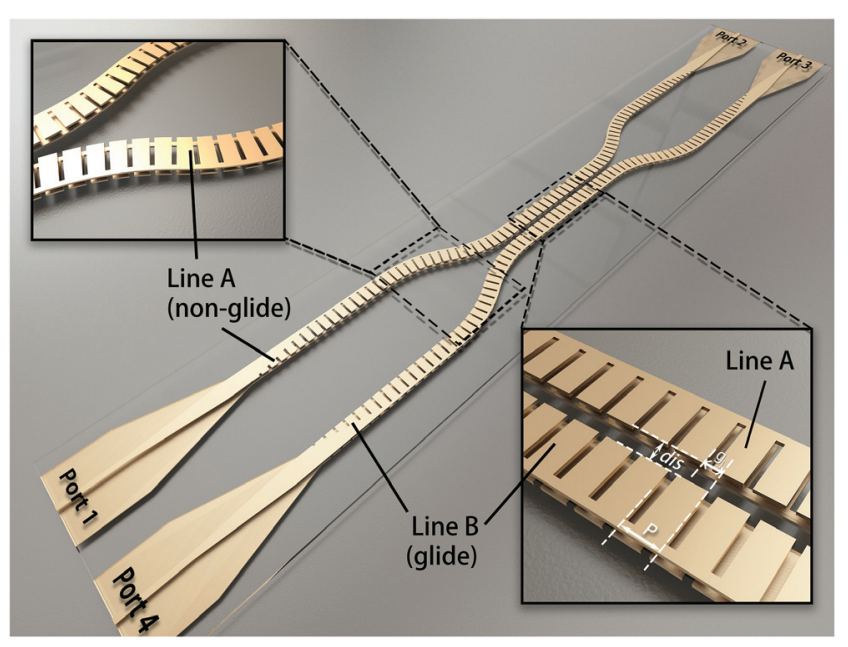

Fig. 6 The four-port model composed of two channels; one is the nonglide symmetric TL channel, and the other is the glide symmetric one.

the coupled mode theory, ${ }^{27}$ in isotropic and homogeneous background medium, when the nonglide TL (line $A$ in Fig. 6) and glide symmetric TL (line $B$ ) are individually excited, the coupling coefficient between them can be expressed as

$\kappa_{a b}=\frac{\omega \varepsilon_{0}}{4} \iint_{s}\left(n^{2}-n_{e a b}^{2}\right)\left[E_{a t}^{*} \cdot E_{b t}+\left(\frac{n_{e a b}^{2}}{n^{2}}\right) E_{a x}^{*} \cdot E_{b x}\right] \mathrm{d} S$,

$\kappa_{b a}=\frac{\omega \varepsilon_{0}}{4} \iint_{s}\left(n^{2}-n_{e b a}^{2}\right)\left[E_{a t}^{*} \cdot E_{b t}+\left(\frac{n_{e b a}^{2}}{n^{2}}\right) E_{a x}^{*} \cdot E_{b x}\right] \mathrm{d} S$,

where $S$ represents the transverse section of TL (the $Y o Z$ plane), and $n$ is the refractive index of the surrounding medium. Since the two TLs are not identical, we use $n_{e a b}$ and $n_{e b a}$ as the TLs' effective refractive indexes when line $A$ and line $B$ are excited, respectively. Note that the SSPPs are TM modes; thus, the longitudinal electric components $\left(E_{a x}\right.$ in line $A$ and $E_{b x}$ in line $B$ ) cannot be neglected when we calculate the mode coupling coefficient. Equations (1a) and (1b) indicate the coupling coefficients to the other TL when the nonglide or glide symmetric TL is individually excited, respectively. In addition, this passive four-port system follows the principle of reciprocity, i.e., no matter which TL is excited, the interline coupling in terms of scattering parameters is identical, as is demonstrated in Fig. 7. With different separations between the two TLs (dis $=0.6$ and $2 \mathrm{~mm}$ ), the isolation coefficients (S14 and S41) and coupling coefficients (S31 and S24) remain the same no matter which SSPP TL is fed. As a result, we have $\kappa_{a b}=\kappa_{b a}$. Putting the electric fields in the fundamental modes of the nonglide and glide symmetric SSPP structures to Eqs. (1a) and (1b), we conclude that $n_{e a b}$ is approximately equal to $n_{e b a}$. In fact, this conclusion is easy to understand because the Brillouin diagrams of the adjacently located glide and nonglide SSPP structures are nearly identical when the two structures are exchanged, indicating the same propagation constants as well as refractive indexes of $n_{e a b}$ and $n_{e b a}$. Therefore, for the two SSPP TLs, the mode coupling coefficient can be represented as

$\kappa_{a b}=\frac{\omega \varepsilon_{0}}{4} \iint_{s}\left(n^{2}-n_{e}^{2}\right)\left[E_{a t}^{*} \cdot E_{b t}+\left(\frac{n_{e}^{2}}{n^{2}}\right) E_{a x}^{*} \cdot E_{b x}\right] \mathrm{d} S$.

Here, $n_{e}$ is the equivalent refractive index of TLs in the array. Next, we use Eq. (2) to compare the coupling coefficient between two identical nonglide symmetric SSPP TLs and that between a nonglide TL and a glide one. $E_{a t}$ and $E_{b t}$ are the transverse electric fields of the fundamental modes in the nonglide and glide symmetric SSPP TLs, respectively, which are calculated in CST using eigenmode simulation; $E_{a x}$ and $E_{b x}$ are the longitudinal ones. The normalized and average values of the mode coupling coefficient are given in Fig. 8. When 


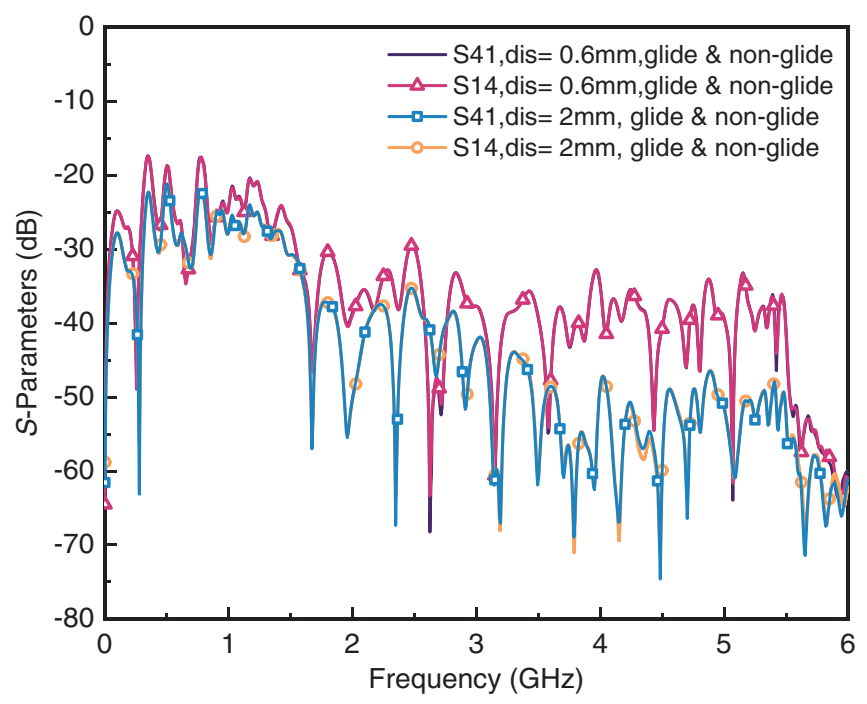

(a)

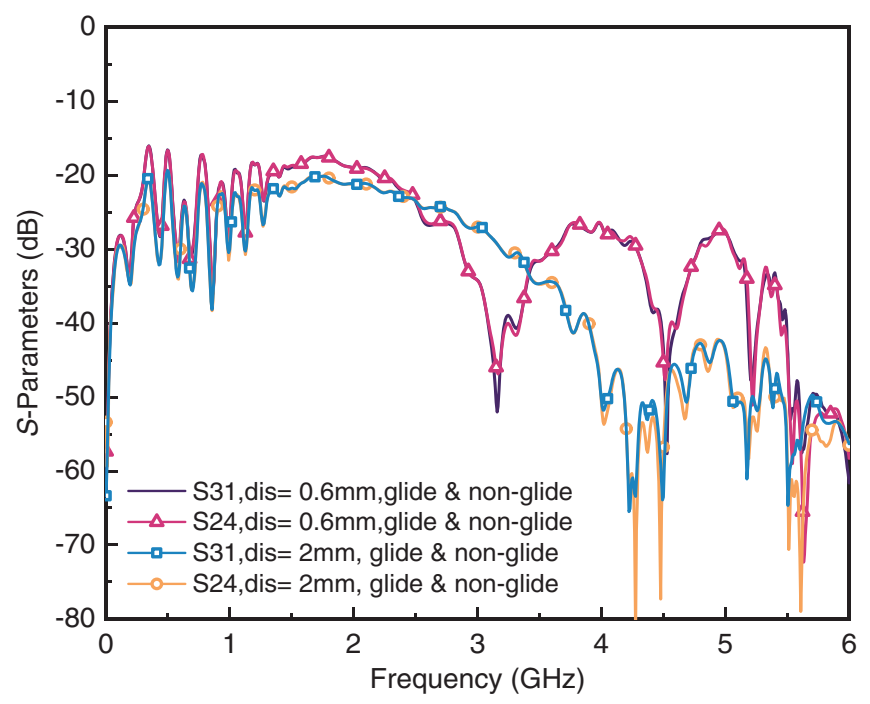

(b)

Fig. 7 Simulated isolation coefficients (S41 and S14) and coupling coefficients (S31 and S24) of the two SSPP TLS.

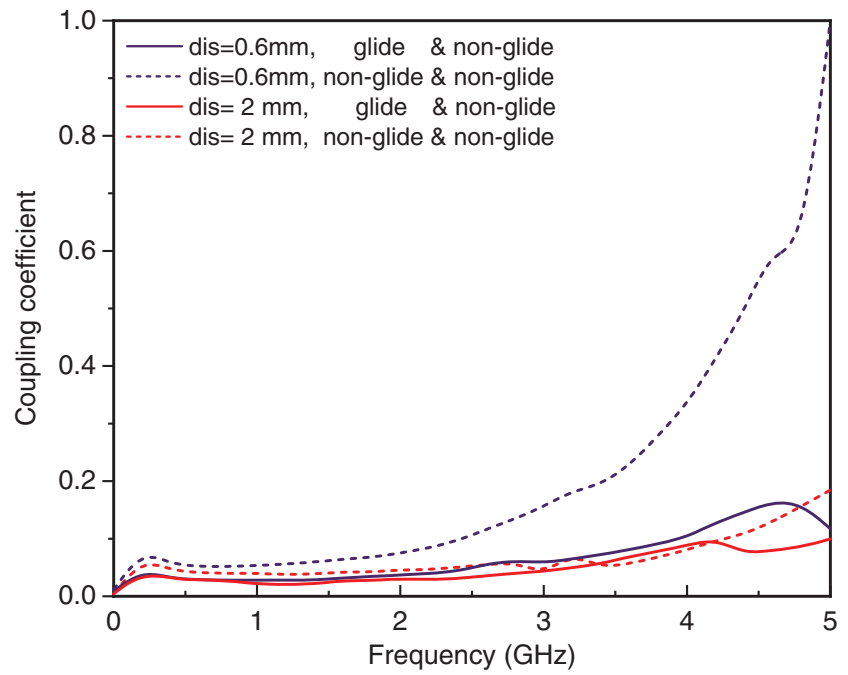

Fig. 8 The normalized and average mode coupling coefficients in two types of TL arrays at different line separations (dis = $0.6 \mathrm{~mm}$ and dis $=2 \mathrm{~mm}$ ).

the separation is $0.6 \mathrm{~mm}$, which is $\lambda_{0} / 100$ at $5 \mathrm{GHz}$, we observe that the coupling coefficient between the glide symmetric and the nonglide symmetric TLs (see the solid black line) is significantly lower than that between two nonglide symmetric TLs (see the dashed black line) from 0 to $5 \mathrm{GHz}$. In particular, as the working frequency approaches the cutoff frequency of $5.4 \mathrm{GHz}$, field localization increases around the SSPP TLs in the subwavelength scale, and thus the dashed black line rises quickly at high frequencies. In contrast, the solid black line is almost flat, indicating that the channel isolation is effectively improved when a nonglide TL is replaced by a glide symmetric one. When the separation is $2 \mathrm{~mm}$, the two mode coupling coefficients (see the solid red line and the dashed red line) become lower and comparable, indicating that the dual-strip SSPP TL array, whether hybrid or uniform, possesses remarkable channel isolation at subwavelength separation due to the significant field confinement of SSPPs.

A prototype of the hybrid array containing nonglide and glide symmetric TLs (as given in Fig. 6) and a control prototype of two uniform nonglide symmetric TLs are fabricated and measured for demonstration. The reflection coefficient S11, transmission coefficient S21, coupling coefficient S31, and isolation coefficient $\mathrm{S} 41$ are measured and plotted in Fig. 9. The four-port networks are fed through the nonglide symmetric TLs at port 1 (see Fig. 6 for reference), and two separations (0.6 and $2 \mathrm{~mm}$ ) are involved in comparisons. In Figs. 9(a) and 9(b), the S11 curves are observed to be similar for the hybrid TL array and the uniform TL array with both separations. When the line separation is $0.6 \mathrm{~mm}$, the S21 curve of the hybrid TL array is higher than that of the uniform TL array from 3.4 to $5.3 \mathrm{GHz}$ in both simulation and measurement results, showing higher transmission in the hybrid TL array, as given in Fig. 9(c). When the line separation is $2 \mathrm{~mm}$, however, both TL arrays have similar transmission performance, as shown in Fig. 9(d). Significantly different coupling coefficients (S31) are shown in Figs. 9(e) and 9 (f), showing that much less energy could be coupled to the neighboring TL in the hybrid array when compared with the uniform nonglide array. In view of this, the employment of glide symmetry helps to suppress the channel crosstalk without bringing extra space and feeding networks. Better isolation in the hybrid TL array is also shown in Figs. 9(g) and 9(h) in term of the $\mathrm{S} 41$ curves. We remark that the crosstalk suppression is mainly due to the hybrid configuration of the TL array that increases the channel isolation. If two glide symmetric TLs, instead of two nonglide ones, are adopted to compose the uniform array, the crosstalk in the TL array is much higher than that of the hybrid one in terms of S31 and S41. In Fig. 9, the simulated S parameters of the uniform glide symmetric TL array are also plotted, showing comparable transmission, reflection, coupling, and isolation coefficients with those of the uniform nonglide symmetric TL array.

The coupling suppression in the hybrid TL array is also investigated in terms of the distributions of near-electric fields, and low-coupling and high-isolation are observed at different 


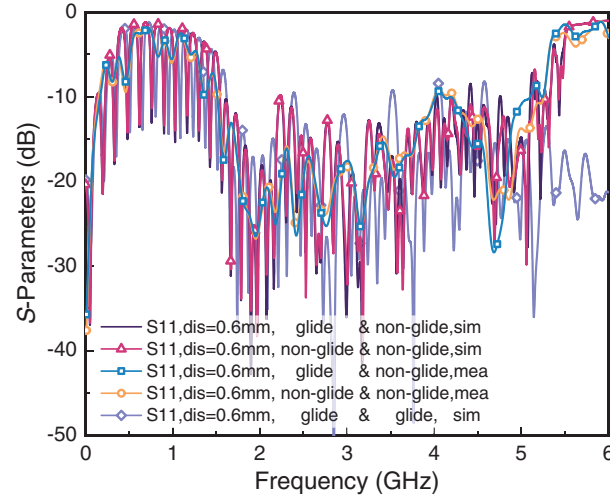

(a)

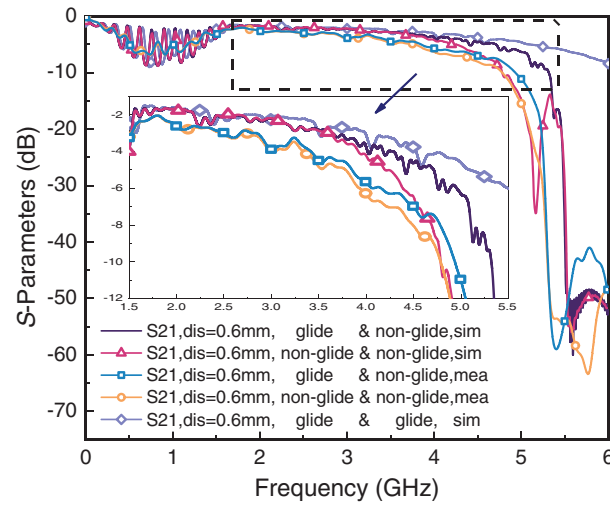

(c)

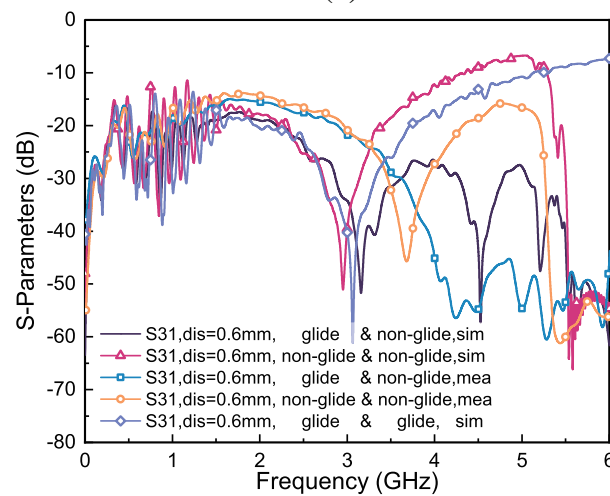

(e)

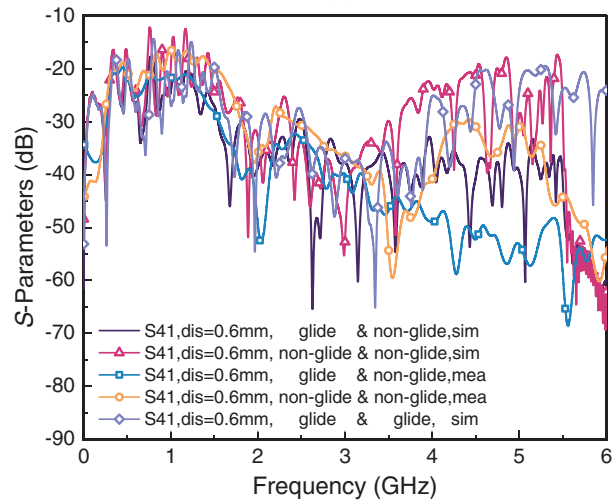

(g)

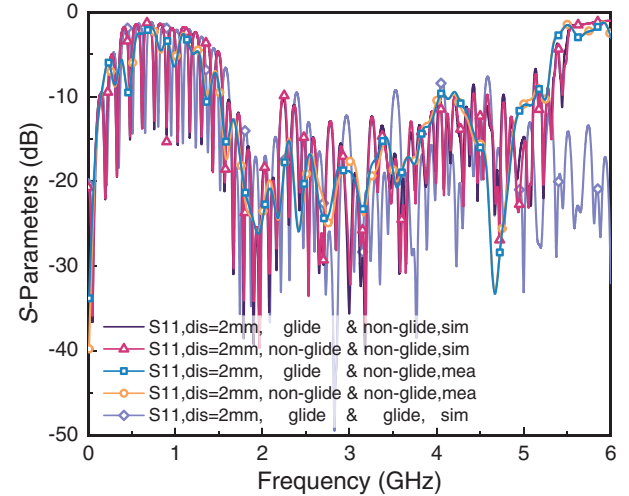

(b)

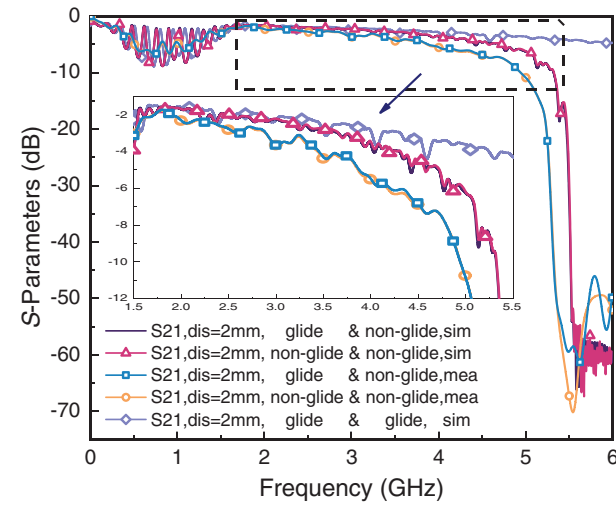

(d)

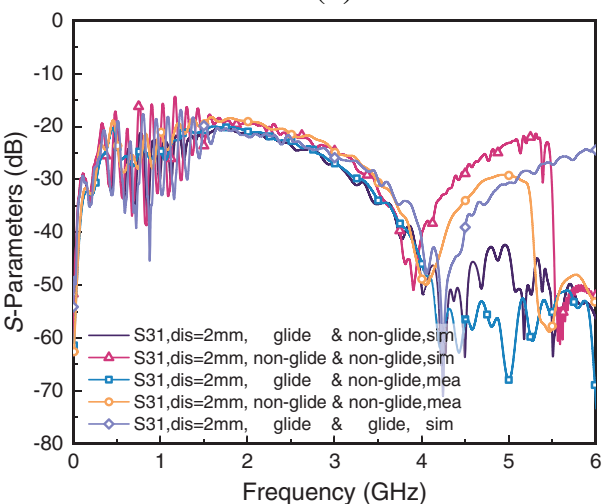

(f)

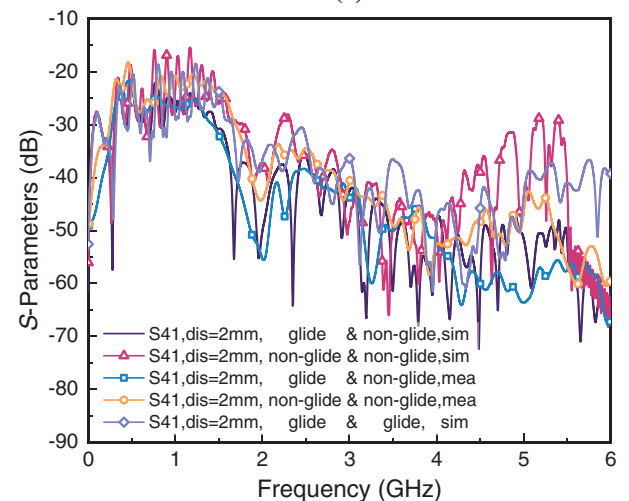

(h)

Fig. 9 Comparison of the reflection coefficient (S11), transmission coefficient (S21), coupling coefficient (S31), and isolation coefficient (S41) for the hybrid TL array (composed of a glide symmetric TL and a nonglide symmetric TL) and the uniform TL arrays (composed of two nonglide symmetric TLs and two glide symmetric TLs, respectively). 
frequencies $(2$ and $5 \mathrm{GHz})$ and with different separations $(0.6$ and $2 \mathrm{~mm}$ ) in both simulation and experiment results, as presented in Fig. 10. Clearly, due to mode mismatch in the hybrid array, very limited portion of energy could be coupled to the neighboring TL and then received at the isolated and coupled ports. This characteristic provides us with a new TL array scheme with low channel crosstalk, improved signal accuracy, and no extra space.

\section{Experimental Setup and Methods}

\subsection{S-Parameter Measurement}

A VNA (Agilent N5230C) was used to perform spectrum measurements of the two-port networks with one SSPP TL and the four-port networks with two SSPP TLs, including all the scattering parameters.
$2 \mathrm{GHz}$, dis $=0.6 \mathrm{~mm}$
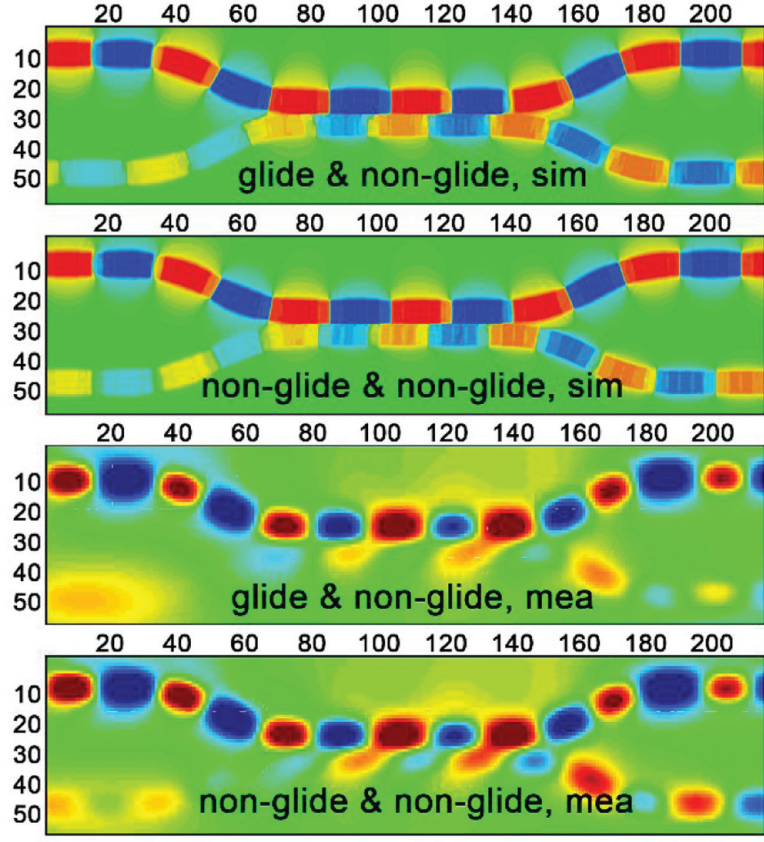

$2 \mathrm{GHz}$, dis $=2 \mathrm{~mm}$

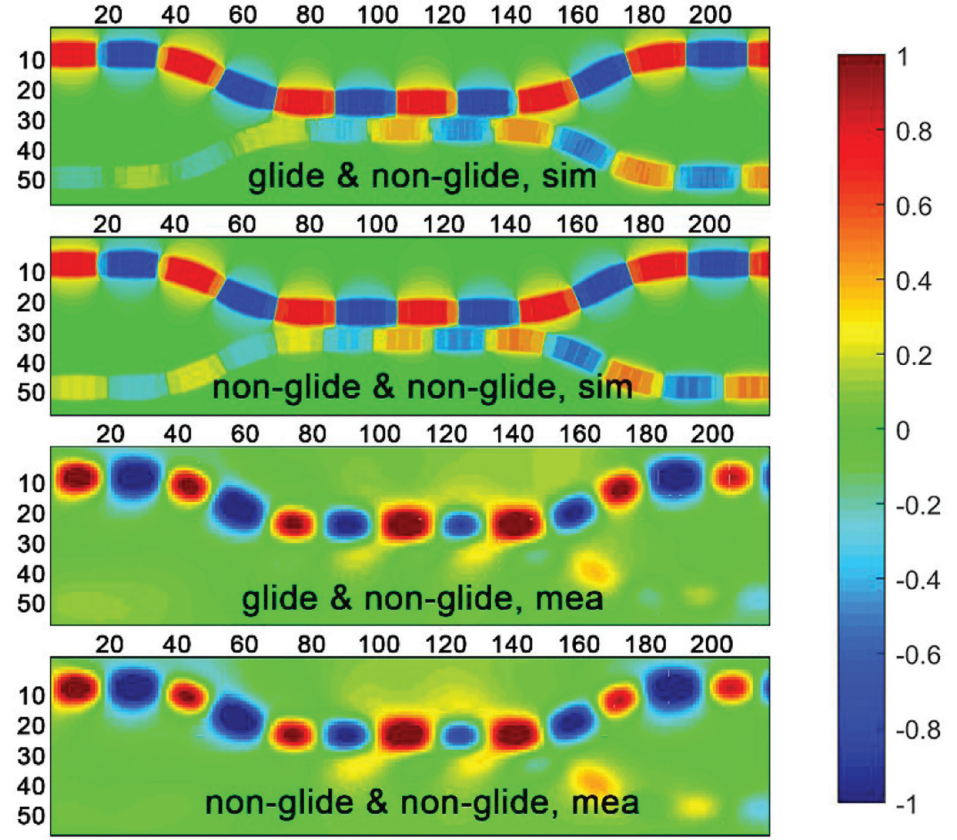

$5 \mathrm{GHz}$, dis $=0.6 \mathrm{~mm}$
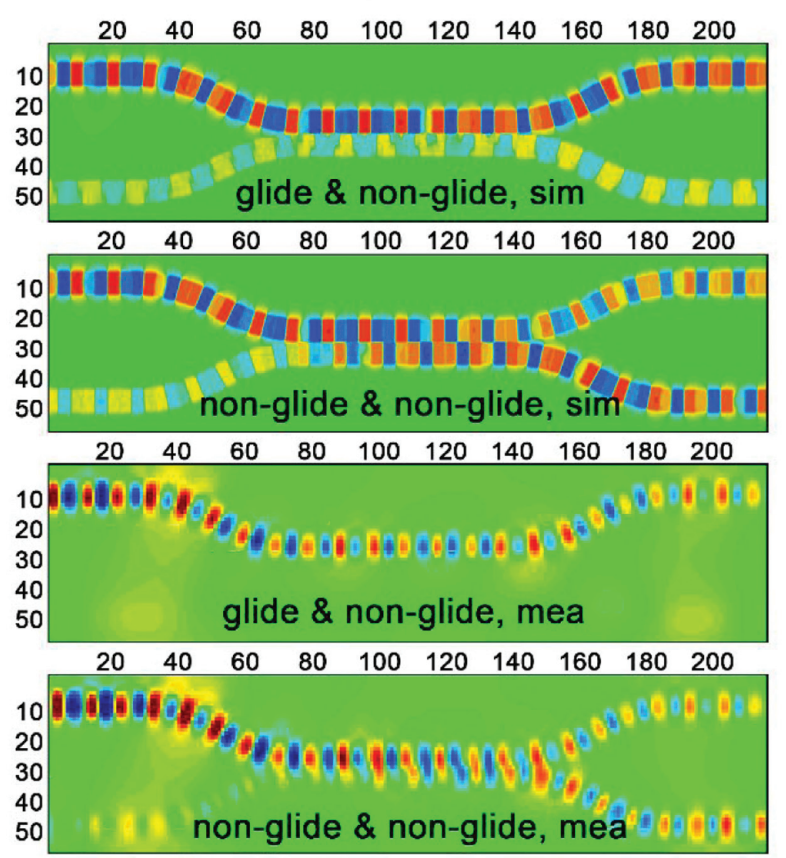

$5 \mathrm{GHz}$, dis $=2 \mathrm{~mm}$

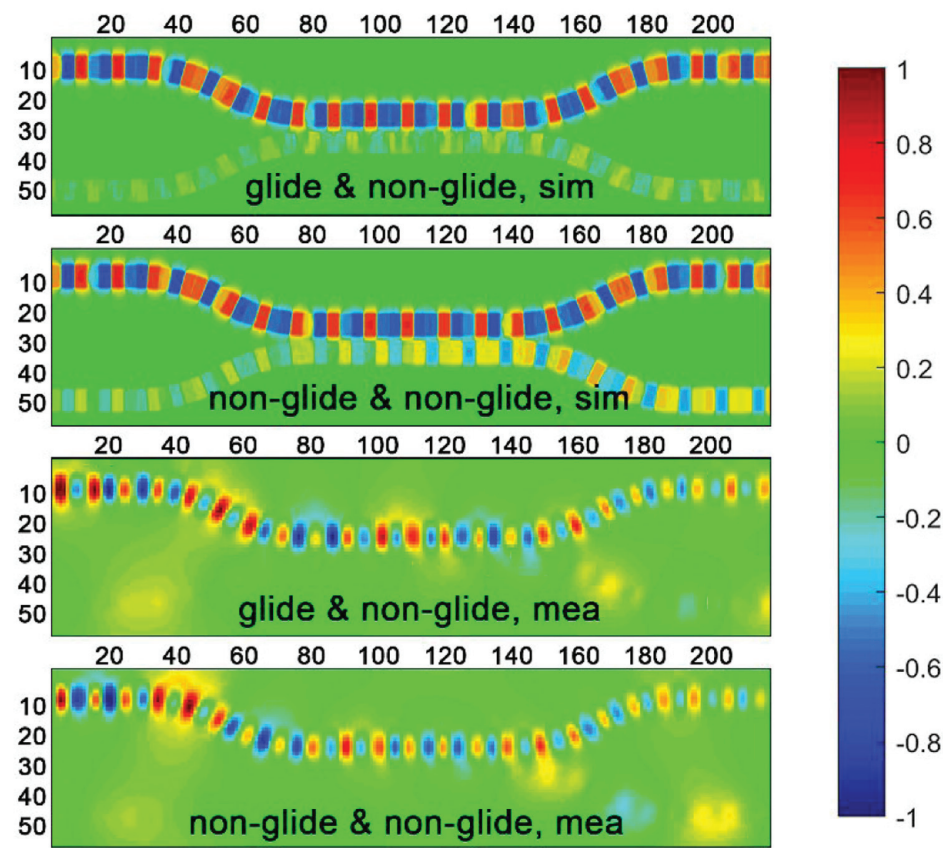

Fig. 10 Distributions of the near-electric field of the hybrid TL array and the uniform TL array with line separations being 0.6 and $2 \mathrm{~mm}$ at 2 and $5 \mathrm{GHz}$, respectively. 


\subsection{Near-Field Measurement}

Near electric field distributions in the SSPP TLs were measured in our home-made 2D near-field scanning system in the microwave anechoic chamber at Southeast University. The 2D nearfield scanning system includes a VNA (Agilent N5230C) and three electronic motor steppers in the $X$-, $Y$-, and $Z$-directions, respectively. The experimental sample was placed on a platform supported by the $X$ - and $Y$-steppers, and a monopole was placed $2 \mathrm{~mm}$ above the sample so as to measure the vertical $(z)$ component of the electric fields continuously when the platform moves in the $\mathrm{XoY}$ plane. The test region of the near fields was $230 \mathrm{~mm} \times 60 \mathrm{~mm}$ (for TL arrays) and $130 \mathrm{~mm} \times 40 \mathrm{~mm}$ (for TLs), and the moving step of the electronic motor was $1 \mathrm{~mm}$.

\section{Conclusion}

In this work, we introduce glide symmetry, which is a typical type of higher symmetry, to manipulate the dispersion characteristics of the SSPP structure. Mode degeneracy and merged pass-bands are investigated and designed so as to broaden the bandwidth and control the propagation modes of SSPPs. We further propose a hybrid SSPP TL array composed of alternately arranged nonglide and glide symmetric SSPP TLs. Although the two neighboring TLs possess the same composing units, strip width, and feeding structure, mutual coupling has been significantly suppressed due to the mode mismatch introduced by the glide symmetry in the structure. Both simulations and experiments have been carried out for demonstration. This glide symmetric SSPP TL, as well as the hybrid array, can be adopted in highly compact circuits without bringing in extra space and feeding networks. In view of this, we conclude that glide symmetry offers supplementary control of the SSPP waves, which may bring about new solutions in future integrated plasmonic circuits.

\section{Acknowledgments}

This work was supported in part from the National Natural Science Foundation of China under Grant Nos. 61631007 and 61971134, in part from the 111 Project under Grant No. 1112-05, and in part from the Fundamental Research Funds for the Central Universities under Grant No. 2242020R40079. Xiao Tian Yan and Wenxuan Tang contributed equally to this work.

\section{Disclosures}

The authors declare no conflicts of interest.

\section{References}

1. W. L. Barnes, A. Dereux, and T. W. Ebbesen, "Surface plasmon subwavelength optics," Nature 424(6950), 824-830 (2003).

2. J. B. Pendry, "Mimicking surface plasmons with structured surfaces," Science 305(5685), 847-848 (2004).

3. W. X. Tang et al., "Concept, theory, design, and applications of spoof surface plasmon polaritons at microwave frequencies," Adv. Opt. Mater. 7(1), 1800421 (2019).

4. X. Shen et al., "Conformal surface plasmons propagating on ultrathin and flexible films," Proc. Natl. Acad. Sci. U. S. A. 110(1), 40-45 (2013).

5. H. F. Ma et al., "Broadband and high-efficiency conversion from guided waves to spoof surface plasmon polaritons: broadband and high-efficiency conversion from guided waves to spoof surface plasmon polaritons," Laser Photonics Rev. 8(1), 146-151 (2014).
6. L. Liu et al., "Multi-channel composite spoof surface plasmon polaritons propagating along periodically corrugated metallic thin films," J. Appl. Phys. 116(1), 013501 (2014).

7. W. Zhang et al., "Trapping of surface plasmon wave through gradient corrugated strip with underlayer ground and manipulating its propagation," Appl. Phys. Lett. 106(2), 021104 (2015).

8. H. C. Zhang et al., "Broadband amplification of spoof surface plasmon polaritons at microwave frequencies: amplification of spoof surface plasmon polaritons," Laser Photonics Rev. 9(1), 83-90 (2015).

9. Q. Zhang et al., "A hybrid circuit for spoof surface plasmons and spatial waveguide modes to reach controllable band-pass filters," Sci. Rep. 5(1), 16531 (2015).

10. X. Shen and T. J. Cui, "Planar plasmonic metamaterial on a thin film with nearly zero thickness," Appl. Phys. Lett. 102(21), 211909 (2013)

11. Y. J. Zhou and B. J. Yang, "A 4-way wavelength demultiplexer based on the plasmonic broadband slow wave system," Opt. Express 22(18), 21589-21599 (2014).

12. J. Y. Yin et al., "Endfire radiations of spoof surface plasmon polaritons," Antennas Wireless Propag. Lett. 16, 597-600 (2017).

13. Q. Zhang, Q. Zhang, and Y. Chen, "Spoof surface plasmon polariton leaky-wave antennas using periodically loaded patches above PEC and AMC ground planes," Antennas Wireless Propag. Lett. 16, 3014-3017 (2017).

14. H. C. Zhang et al., "A plasmonic route for the integrated wireless communication of subdiffraction-limited signals," Light Sci. Appl. 9(1), 113 (2020).

15. W. X. Tang et al., "Reduction of radiation loss at small-radius bend using spoof surface plasmon polariton transmission line," Sci. Rep. 7(1), 41077 (2017).

16. A. Kianinejad, Z. N. Chen, and C.-W. Qiu, "Low-loss spoof surface plasmon slow-wave transmission lines with compact transition and high isolation," IEEE Trans. Microwave Theory Tech. 64(10), 3078-3086 (2016).

17. H. C. Zhang et al., "Breaking the challenge of signal integrity using time-domain spoof surface plasmon polaritons," ACS Photonics 2(9), 1333-1340 (2015).

18. Y. Liang et al., "On-chip sub-terahertz surface plasmon polariton transmission lines in CMOS," Sci. Rep. 5(1), 14853 (2015).

19. A. Hessel et al., "Propagation in periodically loaded waveguides with higher symmetries," Proc. IEEE 61(2), 183-195 (1973).

20. P. Padilla et al., "Glide symmetry to prevent the lowest stopband of printed corrugated transmission lines," IEEE Microwave Wireless Comp. Lett. 28(9), 750-752 (2018).

21. F. Ghasemifard, M. Norgren, and O. Quevedo-Teruel, "Twist and polar glide symmetries: an additional degree of freedom to control the propagation characteristics of periodic structures," Sci. Rep. 8(1), 11266 (2018).

22. O. Quevedo-Teruel, M. Ebrahimpouri, and M. Ng Mou Kehn, "Ultrawideband metasurface lenses based on off-shifted opposite layers," Antennas Wireless Propag. Lett. 15, 484-487 (2016).

23. L. Liu et al., "Backward phase matching for second harmonic generation in negative-index conformal surface plasmonic metamaterials," Adv. Sci. 5(11), 1800661 (2018).

24. L. Wang, J. L. Gomez-Tornero, and O. Quevedo-Teruel, "Substrate integrated waveguide leaky-wave antenna with wide bandwidth via prism coupling," IEEE Trans. Microwave Theory Tech. 66(6), 3110-3118 (2018).

25. H. C. Zhang et al., "Second-harmonic generation of spoof surface plasmon polaritons using nonlinear plasmonic metamaterials," ACS Photonics 3(1), 139-146 (2016).

26. G. Valerio et al., "Glide-symmetric all-metal holey metasurfaces for low-dispersive artificial materials: modeling and properties," IEEE Trans. Microwave Theory Tech. 66(7), 3210-3223 (2018).

27. A. Ma, Y. Li, and X. Zhang, "Coupled mode theory for surface plasmon polariton waveguides," Plasmonics 8(2), 769-777 (2013). 
Xiao Tian Yan received his BS degree from the School of Electronic and Optical Engineering from Nanjing University of Science \& Technology (NUST), Nanjing, China, in 2016. He is currently pursuing his MS degree with the State Key Laboratory of Millimeter Waves, School of Information Science and Engineering, Southeast University, Nanjing, China. His current research interests are millimeter wave plasmonic circuits and their applications on wafers.

Wenxuan Tang received her BSc and MSc degrees from Southeast University, Nanjing, China, in 2006 and 2009, respectively, and her $\mathrm{PhD}$ in electromagnetics from Queen Mary University of London, London, United Kingdom, in 2012. In 2012, she joined the School of Information Science and Engineering, Southeast University, where she is currently an associate professor with the State Key Laboratory of Millimeter Waves. She has authored or coauthored over 30 journal articles, one book, and two book chapters on metamaterials and their applications. Her current research interests include metamaterials and their applications, and microwave devices and antennas. She is an associate editor of Advanced Electromagnetics (AEM) and a guest editor of the Journal of EPJ Applied Metamaterials.

Jun Feng Liu received his BS degree in electromagnetic waves and microwave technology from the University of Electronic Science and Technology of China, Chengdu, China, in 2012 . He is currently pursuing his $\mathrm{PhD}$ in microwave engineering with Southeast University, Nanjing, China. His research interests include the design of metamaterials, spoof surface plasmon polaritons, and antennas.

Meng Wang received his BS degree from NUST, Nanjing, China, in 2015. $\mathrm{He}$ is currently pursuing his $\mathrm{PhD}$ at Southeast University, Nanjing. His current research interests include array antennas, leaky-wave antennas, and spoof surface plasmon polaritons. He was a recipient of the Best
Student Paper Award at the 2018 International Conference on Microwave and Millimeter Wave Technology.

Xin Xin Gao received her MS degree from Nanjing University of Aeronautics and Astronautics of China, Nanjing, China, in 2018. She is currently pursuing her PhD at Southeast University, Nanjing, China. Her research interests include surface plasmonic polaritons, metamaterials, and millimeter wave theories and technologies.

Tie Jun Cui received his BSc, MSc, and PhD degrees in electrical engineering from Xidian University, Xi'an, China, in 1987, 1990, and 1993, respectively. In 1993, he joined the Department of Electromagnetic Engineering, Xidian University, and was promoted to an associate professor in 1993. From 1995 to 1997, he was a research fellow with the Institut fur Hochstfrequenztechnik und Elektronik, University of Karlsruhe, Karlsruhe, Germany. In 1997, he joined the Center for Computational Electromagnetics, Department of Electrical and Computer Engineering, University of Illinois at Urbana-Champaign, Champaign, IL, USA, first as a post-doctoral research associate and then as a research scientist. In 2001, he became Cheung-Kong Professor with the Department of Radio Engineering, Southeast University, Nanjing, China, where he is currently the Chief Professor. He has authored Metamaterials: Theory, Design, and Applications (Springer, 2009) and Metamaterials: Beyond Crystals, Noncrystals, and Quasicrystals (CRC Press, 2016). He has authored or coauthored over 500 peer-reviewed journal articles, which have been cited more than 33,000 times (h-factor 90 ). His research has been selected as one of the "Optics in 2016" by Optics and Photonics News Magazine (OSA), 10 Breakthroughs of China Science in 2010, "Best of 2010" in the New Journal of Physics, and Research Highlights in Europhysics News, Journal of Physics D: Applied Physics, Applied Physics Letters, and Nature China. His work has been reported by Nature News, Science, MIT Technology Review, Scientific American, and New Scientists. 